\title{
CDISC SEND Implantation Findings Result Category Terminology
}

National Cancer Institute

\section{Source}

National Cancer Institute. CDISC SEND Implantation Findings Result Category

Terminology. NCI Thesaurus. Code C124316.

Terminology associated with the implantation findings result category codelist of the Clinical Data Interchange Standards Consortium (CDISC) Standard for the Exchange of Non-clinical data (SEND). 